experience. The mode of administration recommended by Stricker is that twenty to thirty grains be given every hour for six doses, but at the first trial at St. Mary's only seven grains and a half were given at each of the six hours, simply suspended in water. No bad effects being observed, the dose was increased. It was impossible not to be astonished with the effects, and notwithstanding the many disillusions experience in medicine brings, not a few of which have been furnished by acute rheumatism, I should not do justice to my conviction were I not to say that apparently we have in salicylic acid, as Dr. Maclagan has said in bis communication, a remedy for rheumatic fever comparable to quinine as a remedy for ague. According to present experience rheumatic fever when treated by this drug is an affair of two or three days. The disease is common enough, and its usual course sufficiently well known, so that no long time will be required to establish some definite conclusion, and to bring out any possible injurious effects. The only complaint hitherto made of the acid is that it is hot and irritating to the throat; given in milk vomiting has been produced.

Careful examination of the effects on the pulse, temperature, urine, \&c., will no doubt yield important information. Mr. Sworder, who has watched the cases very closely, states that the temperature invariably rises for a short time after the administration of the first dose, but the observations recorded in the careful notes taken at short intervals by him and Mr. Gawith show a gradual fall both of temperature and pulse rate. No sphygmograpic observations were made. Relief from pain was always quickly obtained, and, as a rule, the patients slept well, no opiate being required; as a rule, again, there was very free perspiration, but this of course is common in acute rheumatism.

\section{ROYAL FREE HOSPITAL}

STONE IN THE BLADDER IN A YOUNG CHILD; LATERAI LITHOTOMY ; RECOVERY.

(Under the care of Mr. WILLIam Rose.)

THene has lately been a distinct revival of interest in the subject of stone in the bladder. The report of the series of cases from the Leicester Infirmary which we published three weeks ago, presented an interesting record as far as it went of two years' work at that hospital. It may be remembered that at Leicester the median operation was performed in every instance, with one death out of twenty operations. Such a statement could not, of course, be taken as of abso. lute value as regards the average mortality after the median operation, or as determining definitely the comparative merits of that procedure, inasmuch as thirteen of the cases were in children, in whom lithotomy is by no means a dan gerous operation. As we understood the record referred to, Mr. Hunt wished, without prejudice, to place before the profession a brief record of the cases of lithotomy treated in the Leicester Infirmary during two years, and that he did satisfac torily. In this connexion the subjoined notes may be of $i_{\text {nterest:- }}$

The patient in this instance was a healthy-looking, wellnourished little boy aged four years, who was admitted into the Royal Free Hospital on March 7th, 1876, suffering from the ordinary symptoms of stone in the bladder. As will be seen hereafter, the stone was unusually large, con sidering $t$ be age of the patient; so large, indeed, that Sir William Fergusson, who was present at the operation, ex pressed the opinion that he had rarely seen so big a stone in so young a subject.

On March 6 th the boy had been taken to the out-patient department. Stone in the bladder was suspected, and sound was passed at once into the bladder. The instrument struck a bard substance which emitted a clear sharp note. There was a small umbilical hernia, but no prolapse of the rectum, and the boy had been circumcised two month before on account of phimosis. The boy was admitted next day, and on March 15th, after a week's rest in bed to allay, as much as possible, the vesical irritation, Mr. Rose performed the usual operation of lateral lithotomy, the staff being held by Mr. Henry Smith. On introducing the forceps the stone was happily caught by its short axis with the first gush of urine and carefully extracted. The calculus, which was a flat ovoid, and a specimen of pure uric acid, weighed when dry 170 grains, and measured $1 \frac{1}{4}$ in. by 1 in.; thickness, $\frac{5}{8}$ in.
We are glad to learn that the patient hss recovered with. out a bad symptom. On the second day the urine ceased to come by the wound, which had quite healed on the fourteenth day from the operation.

\section{SUNDERLAND INFIRMARY.}

CASE OF HYDATIDS OF THE LUNG AND GALL-BLADDER,

(Under the care of Dr. BowMAN.)

THts case is interesting in several particulars-first, as regards the size of the cyst in the interior of the gall-bladder; secondly, the state of the left lung, which pressure had rendered entirely useless, with so little constitutional disturbance; thirdly, as regards the danger in tapping the chest with the pneumatic aspirator; fourthly, the sudden termination of the case in a man, to all appearance, in good health.

Ed. R-, aged twenty-nine, labourer, was admitted on April 1st, 1874. There was nothing particular to notice in his family history, there being no reason to assign any heredity as a cause of patient's present illness.

For the last five or six years he had complained of shortness of breath upon exertion, and had had a winter cough. He had never been abroad, and had had up to the present attack very good health, with the exception above mentioned. Two years and a half before be first noticed a swelling about the size of half an orange just below the ribs on the right side, which caused pain whenever he felt ont of health. This swelling, he stated, had gradually increased in size. About fourteen months ago he attended at the dispensary, and upon his second visit complained of pain in left side, shortness of breath, cough, and slight expectoration. At this time there was distinct pleuritic friction-sound heard over left lung, but accompanied with very slight dulness. The tumour over the seat of the liver was very distinctly felt, but there was no pain or tenderness. He was advised to become an in-patient of the infirmary, but from this time until admission was not again seen.

On admission, as he was seen in bed, he appeared to be in good health; tongue clean, pulse regular temperature natural, appetite good, bowels regular. Upon examination. the left side of the chest was dull upon percussion, respira. tion almost absent, and voice-sounds nil. This side was slightly larger than the right, although he was righthanded. He complained of no pain, and only of shortness of breath upon exertion. There was present a tumour about the size of a child's head in the region of the gallbladder, globular, fluctuating, painless, and elastic; there were no signs of jaundice, and never had been; he had never received any injury, and could assign no cause for the swelling. All the other organs appeared healthy He was ordered medicine, but as he received no benefit and the tumour had increased in size, eight ounces of fluid were removed by the aspirator on April 19th. The fluid was of a clear colour, and under the microscope a number of hooklets were easily discovered.

April 22nd.-Slight pain after tapping, which soon disappeared. The tumour smaller than on 19 th.

$24 t h$. - As the tumour in the abdomen still continued to diminish, it was considered desirable to tap the chest, which accordingly was done with the aspirator, and four ounces of fluid were withdrawn, when he complained of difficulty of breathing and great pain, but it passed off in a few seconds but the next time the tap was turned, and before two ounces of fiuid had been withdrawn, he was sejz $\gg$ with intense pain and difficulty of breathing; the skin became blue frothy fluid mixed with blood came from the mouth, and in two minutes be fell back dead. Artificial respiration and galvanism were used, but without any signs of retarning animation.

Post.mortem examination.-With the exception of the left lung, the gall-bladder, and the surface of the peritoneum just over it, all the other organs of the body were healthy. Upon opening the chest the left side was nearly filled with fluid, and the heart was pushed over to the right side. Upon removing the fluid the cavity seemed to be empty, but after some difficulty the remains of the lung-tissue were removed, and it was then found that an bydatid cyst had evidently commenced to grow from the centre of the lung, as the 